\title{
Aggregation vs. breakup of the organic phase complex
}

\author{
Sanjeev Kumar*, G.L. Tulasi ${ }^{1}$ \\ Department of Chemical Engineering, Indian Institute of Science, Bangalore 560 012, India
}

Received 16 August 2004; received in revised form 2 November 2004; accepted 11 December 2004

\begin{abstract}
Extraction of zinc and other metals from aqueous solutions using D2EHPA as an extractant in organic phases has been investigated extensively in the past. Under the conditions of low loading of metal in organic phase, equilibrium behaviour is adequately explained through an interfacial reaction with constant stoichiometric coefficients. Experiments show that as the loading of metal in the organic phase increases, increasingly smaller number of molecules of D2EHPA are required to extract a metal ion. Reaction mechanisms which explain this behaviour through breakup of complexes formed on the interface to release free extractant for further extraction are shown to be untenable as they fail to explain experimental observations on viscosity variation and dependence of apparent equilibrium constant on loading ratio alone. A new reaction mechanism is proposed in this work. It considers that complex formed on the interface aggregates in the organic phase and releases free extractant for increased extraction. The proposed mechanism successfully explains the above hitherto unexplained features of metal-D2EHPA extraction systems. It also quantitatively predicts the extraction equilibria not just for zinc but also for cobalt and nickel, and may characterize other extraction systems as well.

(C) 2005 Elsevier B.V. All rights reserved.
\end{abstract}

\section{Introduction}

Extraction of zinc and other metals using extractant D2EHPA (di-2-ethylhexyl phosphoric acid) has been studied extensively in the literature due to the commercial importance of these systems (Lo et al., 1983) and the adoption of Zn-D2EHPA system by the European Federation of Chemical Engineers as

\footnotetext{
* Corresponding author.

E-mail address: sanjeev@chemeng.iisc.ernet.in (S. Kumar).

${ }^{1}$ Current address: GE, JFWTC, Whitefield, Bangalore 560066.
}

the reactive liquid-liquid test system for evaluating performance of extraction equipment (Slater, 1987). The equilibrium behaviour of these extraction systems, characterized through the following type of interfacial reaction

$M^{m+}+q \overline{(H R)_{2}} \stackrel{K_{i}}{\rightleftharpoons}\left(\overline{M R)_{m}(H R)_{2 q-m}}+m H^{+}\right.$,

has subsequently come under renewed focus of researchers. The species $M^{m+}$ and $H^{+}$, confined to aqueous phase, represent metal ion and the hydrogen ion, respectively. The species $\overline{(H R)_{2}}$ and $\overline{M R_{m}(H R)_{2 q-m}}$, confined to the organic phase (indicated by overbar), 
represent the dimer of D2EHPA and the metalD2EHPA complex, respectively.

The value of stoichiometric coefficient $q$ in the above reaction is the focus of recent research activity in this area. Different investigators have used different values of $q$ for the same system. In some cases, if the experiments were carried out over a large concentration range, no single value of $q$ could be found to fit the experimental data. As the concentration of metal-D2EHPA complex in organic phase increases (denoted by $\bar{M}$ ), a lower value of $q$ fits the data better. The equilibrium constant estimated using a constant value of $q$ can thus be called as an apparent equilibrium constant $\left(K_{\mathrm{a}}\right)$ as its value keeps changing. While many parameters affect $K_{\mathrm{a}}$, it is interesting that its dependence on them can be represented through a single variable-loading ratio $L$ (Komasawa et al., 1981; Huang and Juang, 1986), defined as ratio of molar concentration of metalD2EHPA complex to the initial concentration of the extractant. Thus, $K_{\mathrm{a}}$ values have been plotted against $L$ in the literature. The range of loading ratio over which the value of $K_{\mathrm{a}}$ remains the same as its value in limit $L \rightarrow 0$ has been taken as the range of applicability of the mechanism.

A decrease in value of $q$ with an increase in $\bar{M}$ has been sought to be explained by many investigators through the formation of additional complexes with smaller number of D2EHPA molecules per metal ion extracted. The initial efforts considered formation of such complexes through additional in- terfacial reactions. It has now become clear that additional interfacial reactions cannot explain the experimental observations. The reaction mechanism being pursued actively now (Thakur, 1998; Mansur et al., 2002; Morais and Mansur, 2004) is that the complex formed on the interface breaks into a smaller one in the organic phase (with a correspondingly smaller value of $q$ ) and releases free extractant for further extraction.

In this communication, we propose an alternative mechanism to explain a decrease in $q$ and several other hitherto unexplained findings. We propose that complexes with smaller value of $q$ are formed when complexes formed on the interface aggregate in the organic phase and release free extractant for further extraction. We also show that the mechanism involving breakup of complexes formed through interfacial reaction in the organic phase is not tenable. We begin with a review of the pertinent experimental features reported in the literature followed by a review of the previous modelling efforts. We then present the new mechanism proposed in this work.

\section{Summary of significant experimental findings}

Concentration of free extractant in organic phase at equilibrium is not easy to measure, however, under low loading conditions, its value can be taken to be the same as its value initially. Several

Table 1

Variation of viscosity of loaded organic phase with addition of extractant D2EHPA for two diluents, dodecane and benzene; adapted from the Figs. 5 and 6 of Kolarik and Grimm (1976)

\begin{tabular}{|c|c|c|c|c|c|c|c|}
\hline \multicolumn{4}{|l|}{$\mathrm{Zn}$} & \multicolumn{4}{|l|}{ Co } \\
\hline \multicolumn{2}{|l|}{ Dodecane } & \multicolumn{2}{|l|}{ Benzene } & \multicolumn{2}{|l|}{ Dodecane } & \multicolumn{2}{|l|}{ Benzene } \\
\hline $\begin{array}{l}\text { Concentration } \\
\text { of D2EHPA } \\
\text { added, M }\end{array}$ & $\begin{array}{l}\mu \mathrm{N} \\
\mathrm{s} / \mathrm{m}^{2} \times 10^{3}\end{array}$ & $\begin{array}{l}\text { Concentration } \\
\text { of D2EHPA } \\
\text { added, M }\end{array}$ & $\begin{array}{l}\mu \mathrm{N} \\
\mathrm{s} / \mathrm{m}^{2} \times 10^{3}\end{array}$ & $\begin{array}{l}\text { Concentration } \\
\text { of D2EHPA } \\
\text { added, M }\end{array}$ & $\begin{array}{l}\mu \mathrm{N} \\
\mathrm{s} / \mathrm{m}^{2} \times 10^{3}\end{array}$ & $\begin{array}{l}\text { Concentration } \\
\text { of D2EHPA } \\
\text { added, M }\end{array}$ & $\begin{array}{l}\mu \mathrm{N} \\
\mathrm{s} / \mathrm{m}^{2} \times 10^{3}\end{array}$ \\
\hline 0 & 4.9 & - & - & 0 & 9.80 & - & - \\
\hline $5.7 \times 10^{-4}$ & 4.3 & $9.2 \times 10^{-4}$ & 3.6 & $4.37 \times 10^{-3}$ & 5.7 & $4.37 \times 10^{-3}$ & 19.1 \\
\hline $7.2 \times 10^{-4}$ & 3.2 & $2.1 \times 10^{-3}$ & 2.7 & $6.7 \times 10^{-3}$ & 3.4 & $6.4 \times 10^{-3}$ & 10.0 \\
\hline $1.1 \times 10^{-3}$ & 1.8 & $4.0 \times 10^{-3}$ & 1.7 & $9.6 \times 10^{-3}$ & 2.2 & $9.6 \times 10^{-3}$ & 2.94 \\
\hline $5.0 \times 10^{-3}$ & 1.5 & $1.0 \times 10^{-2}$ & 0.85 & $1.95 \times 10^{-2}$ & 2.2 & $1.95 \times 10^{-2}$ & 0.75 \\
\hline $1.0 \times 10^{-2}$ & 1.5 & $2.0 \times 10^{-2}$ & 0.72 & $5.0 \times 10^{-2}$ & 2.2 & $5.0 \times 10^{-2}$ & 0.75 \\
\hline- & - & $4.0 \times 10^{-2}$ & 0.72 & - & - & $1.0 \times 10^{-1}$ & 0.75 \\
\hline
\end{tabular}

Viscosities of diluents benzene and dodecane and pure extractant are $0.67,1.45$, and $40 \mathrm{cp}$, respectively. 
investigators have therefore conducted experiments in dilute limit to estimate stoichiometric coefficients and equilibrium constant for interfacial reactions. For the specific case of extraction of $\mathrm{Zn}$, different values of $q$ have been reported in dilute limit. These are now attributed to the nature of the diluent; aliphatics yield a value of 1.5 and aromatics 2.0. Heptane as a diluent has mostly yielded a value of $q$ close to 1.5 (Ajawin et al., 1983; Huang and Juang, 1986; Sainz-Diaz et al., 1996). Huang and Juang (1986) have reported the value of equilibrium constant to be $0.945 \times 10^{-2} \mathrm{M}^{1 / 2}$ for kerosene as solvent and Ajawin et al. (1983) have reported the same to be $0.735 \times 10^{-2} \mathrm{M}^{1 / 2}$ for heptane as solvent. The value of $q$ is reported to decrease from 1.5 (or 2.0) to 1.0 as the concentration of $\mathrm{Zn}-$ D2EHPA complex in the organic phase increases (Kolarik and Grimm, 1976; Bart et al., 1992; Thakur, 1998; Nishihama et al., 1999; Mansur et al., 2002). Similar findings are available for extraction of other metals as well.

Measurements of viscosity of the loaded organic phase, reported by Kolarik and Grimm (1976), show it to increase significantly (as much as 20 times) with an increase in loading of $\mathrm{Zn}$ and $\mathrm{Co}$ in two diluents, dodecane and benzene. Addition of a small amount of extractant however decreases the viscosity of the loaded organic phase dramatically in all the cases. These measurements are summarized in Table 1. Kolarik and Grimm explain this behaviour through the formation of very large complexes in the loaded organic phase, which are broken when free extractant is added to it.

\section{Review of modelling approaches}

A large number of investigators have considered decrease in $q$ to be caused by the formation of complexes with smaller number of D2EHPA molecules per metal ion, and these are reviewed here. Bart et al. (1992) have considered $q$ to change due to the nonideal behaviour of highly loaded organic phase. The readers can refer to Mansur et al. (2002) for a review of this approach.

Brisk and McManamey (1969) have proposed that the complex formed on the interface through the reaction between metal and D2EHPA combines with more metal through a second interfacial reaction to make a bigger complex with less number of D2EHPA molecule per metal ion:

$$
\left.\begin{array}{l}
\frac{M^{2+}+2(\overline{H R})_{2}}{\left(M R_{2}\right)(H R)_{2}}+\overline{(H R)_{2}}+M^{2+} \rightleftharpoons \frac{\overline{M R_{2}(H R)_{2}}+2 H^{+}}{\left(M R_{2}\right)_{2}(H R)_{2}}+2 H^{+}
\end{array}\right\}
$$

Nishihama et al. (1999) have proposed a similar mechanism, but with a different interfacial reaction to form bigger complexes, as shown below.

$$
\left.\begin{array}{l}
Z^{2+}+2 \overline{(H R)_{2}} \\
Z n^{2+}+\overline{Z n R_{2}(H R)_{2}} \rightleftharpoons \overline{Z n R_{2}(H R)_{2}}+2 H^{+} \\
\overline{Z n_{2} R_{4}}+2 H^{+}
\end{array}\right\}
$$

As both of these mechanisms propose all the reactions to be interfacial reactions, interconversion between two complexes cannot occur in the absence of an interface, and hence, they are unlikely to explain the rapid decrease in viscosity, thought to be due to the interconversion of bigger complexes into smaller ones (Kolarik and Grimm, 1976). Thakur (1998) has also proposed two interfacial reactions but these proceed in parallel.

$\left.\begin{array}{l}M^{2+}+2 \overline{(H R)_{2}} \stackrel{\stackrel{K_{1}}{\rightleftharpoons} \overline{M R_{2}(H R)_{2}}+2 H^{+}}{M^{2+}+\overline{(H R)_{2}} \stackrel{K_{2}}{\rightleftharpoons} \overline{M R_{2}}+2 H^{+}}\end{array}\right\}$

He however permits interconversion between two complexes through

$\overline{M R_{2}}+\overline{(H R)_{2}} \stackrel{K_{3}}{\rightleftharpoons} \overline{M R_{2}(H R)_{2}}$

with $K_{3}$ same as $K_{1} / K_{2}$. The above reaction proceeds in the bulk of the organic phase, and is perhaps the first one to consider breakup of complex formed on the interface in the bulk to release free extractant.

Mansur et al. (2002) have proposed a two-step reaction mechanism, shown below. The first reaction is the same as the interfacial reaction proposed by Ajawin et al. (1983), Huang and Juang (1986), and Sainz-Diaz et al. (1996) to explain equilibrium data under low loading conditions. The second reaction is the breakup of the complex formed on the interface, as per the mechanism of Thakur (1998), and is expected 
to play a role at high loading of metal in the organic phase.

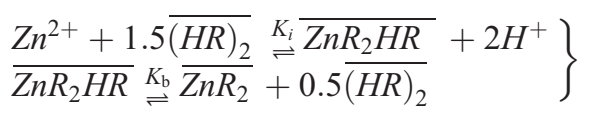

Breakup of complex at high loading suggests that viscosity of the organic phase should decrease with an increase in the loading of metal (assuming that the complex formed on breakup is of nearly the same size as the dimer of the extractant). Addition of free extractant to loaded organic phase should lead to formation of bigger complexes through backward reaction in the bulk, and hence an increase in viscosity of the organic phase. Both of these are not in agreement with the experimental findings discussed earlier. Morais and Mansur (2004), while commenting on this mechanism (to be referred to as breakup based mechanism), state that the breakup of complex plays an insignificant role at low loading conditions, hence interfacial reaction alone is capable of explaining the experimental data. We will later see that even in the limit of zero loading of metal in the organic phase, breakup of complexes can determine equilibrium extraction behaviour. Morais and Mansur have provided spectroscopic data which according to them supports formation of smaller complexes at high loading. We will revisit this data later to examine if it unequivocally permits such an interpretation.

Thus, to summarize, the equilibrium extraction behaviour of zinc-D2EHPA and other similar systems is yet not fully understood. There is a need to explain the experimental findings discussed above along with the equilibrium data in a consistent manner.

\section{A new mechanism for extraction of $\mathrm{Zn}$ and other metals}

When aqueous and organic phases containing metal ion $M^{m+}$ and extractant $(\mathrm{HR})_{2}$ (denoted by $\overline{\mathrm{B}}$ ), respectively are contacted, the two react with each other at the interface to form metal-D2EHPA complexes through the following reaction

$M^{m+}+q \overline{(H R)_{2}} \stackrel{K_{i}}{\rightleftharpoons} \overline{M R_{2}(H R)_{2 q-m}}+m H^{+}$

which is the same as that proposed by the earlier investigators (Komasawa et al., 1981; Huang and
Juang, 1986; Mansur et al., 2002) to explain experimental data under low loading conditions. We now propose that complex $\overline{M R_{2}(H R)_{2 q-m}}$ (denoted as $\overline{\mathrm{C}}$ ) formed on the interface further reacts (aggregates) with itself in the bulk of the organic phase to form larger complex $\left(M_{2}(2 q-m-1) H R\right)_{2}$ (denoted as $\overline{\mathrm{D}})$ and releases some carrier, which can extract additional metal ions through interfacial reaction, thereby allowing for more efficient utilization of carrier. This reaction mechanism is similar to the mechanism proposed earlier by us (Tulasi and Kumar, 1999) to explain the equilibrium extraction behaviour of amino acids using D2EHPA. Thus,

$\bar{C}+\bar{C} \stackrel{K_{\mathrm{b}}}{\rightleftharpoons} \bar{D}+\bar{B}$

Here,

$K_{i}=\frac{[\bar{C}]\left[H^{+}\right]^{m}}{\left[M^{m+}\right][\bar{B}]^{q}}$

and

$K_{\mathrm{b}}=\frac{[\bar{D}][\bar{B}]}{[\bar{C}]^{2}}$

The complexes $\overline{\mathrm{C}}$ and $\overline{\mathrm{D}}$, respectively have $q$ and $(q-1 / 2)$ dimers of D2EHPA per metal ion.

Let us now define an apparent equilibrium constant $K_{\mathrm{a}}$ for the interfacial reaction. While $K_{i}$ is based on actual concentrations of the species (Eq. (9)), $K_{\mathrm{a}}$ is based on apparent concentration of species $\overline{\mathrm{B}}$ and $\overline{\mathrm{C}}$, arrived at by assuming that $\overline{\mathrm{C}}$ does not aggregate. Thus,

$K_{\mathrm{a}}=\frac{[\bar{C}]_{\mathrm{a}}\left[H^{+}\right]^{m}}{\left[M^{m+}\right][\bar{B}]_{\mathrm{a}}^{q}}$

where $[\overline{\mathrm{C}}]_{\mathrm{a}}$ is the same as $[\overline{\mathrm{M}}]$, the total concentration of solute in organic phase in the form of complexes $\overline{\mathrm{C}}$ and $\overline{\mathrm{D}}$, and $[\overline{\mathrm{B}}]_{\mathrm{a}}$ is $[\overline{\mathrm{B}}]_{\mathrm{i}}-[\overline{\mathrm{M}}]$. Mass balances for metal ions and carrier in the organic phase yield:

$$
[\bar{M}]=[\bar{C}]+2[\bar{D}]
$$

$[\bar{B}]=[\bar{B}]_{i}-(q[\bar{C}]+(2 q-1)[\bar{D}])$ 
We now formally define loading ratio $L$ and free carrier ratio $r$ as

$L=\frac{[\bar{M}]}{[\bar{B}]_{i}}$

$r=\frac{[\bar{B}]}{[\bar{B}]_{i}}$

The concentrations $[\overline{\mathrm{C}}]$ and $[\overline{\mathrm{D}}]$ appearing in Eqs. (12) and (13) cannot be measured experimentally but are known in terms of other measurable concentrations through Eqs. (9) and (10). Thus, combining Eqs. (12) and (13) with Eqs. (9) and (10) and introducing concentration ratios $L$ and $r$ in them, we obtain the following final expressions for $r$ and $K_{\mathrm{a}}$.

$$
r=1-K_{i} \frac{q L r^{q}}{(1-q L)^{q} K_{\mathrm{a}}}-K_{\mathrm{b}}\left(\frac{K_{i}}{K_{\mathrm{a}}}\right)^{2} \frac{(2 q-1) L^{2} r^{2 q-1}}{(1-q L)^{2 q}}
$$

$\frac{K_{\mathrm{a}}}{K_{i}}=\frac{r^{q}}{(1-q L)^{q}}+2 K_{\mathrm{b}}\left(\frac{K_{i}}{K_{\mathrm{a}}}\right) \frac{L r^{2 q-1}}{(1-q L)^{2 q}}$

Eqs. (16) and (17) together make a system of coupled algebraic equations with three variables $r, K_{\mathrm{a}}$, and $L$.
For given values of true equilibrium constants $K_{i}$ and $K_{\mathrm{b}}$, the variation of $K_{\mathrm{a}}$ and $r$ with $L$ can be obtained by solving these equations numerically. As no other variable appears in Eqs. (16) and (17), the proposed mechanism can be taken to explain the experimental observation that $K_{\mathrm{a}}$ depends on $L$ alone. In the asymptotic limit of $L \rightarrow 0$, the above model equations simplify to

$K_{\mathrm{a}}=K_{i}\left(1+2 K_{\mathrm{b}} L\right)$

$r=1-q L$

$[\tilde{\bar{C}}]=L\left(1-2 K_{i} K_{\mathrm{b}} L\right)$

$[\tilde{\bar{D}}]=K_{\mathrm{b}} L^{2}$

$[\tilde{\bar{C}}]$ and $[\tilde{\bar{D}}]$ are normalized concentrations with respect to $[\overline{\mathrm{B}}]_{\mathrm{i}}$. The above asymptotic solution, which matches with the exact solution for $L$ values up to 0.2 , shows an interesting feature. As $L \rightarrow 0,[\overline{\mathrm{C}}]$ varies with $L$ linearly while $[\overline{\mathrm{D}}]$ varies quadratically. This indicates that the proposed reaction in the bulk does

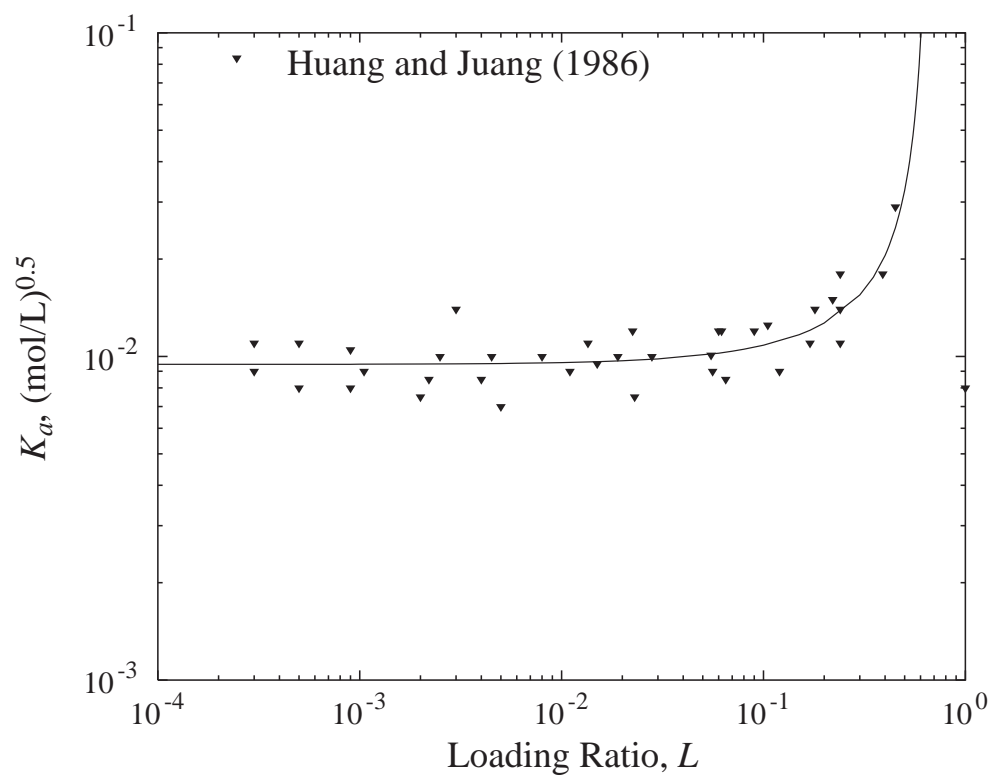

Fig. 1. Apparent equilibrium constant vs. loading ratio $L$ for Zn-D2EHPA system for the data of Huang and Juang (1986), $m=2, q=1.5$, $K_{i}=0.945 \times 10^{-2} \mathrm{M}^{1 / 2}, K_{\mathrm{b}}=0.65$. 
Table 2

Stoichiometric coefficients and equilibrium constants

\begin{tabular}{llllll}
\hline Metal & $m$ & $q$ & $K_{i}$ & Ref. & $K_{\mathrm{b}}$ \\
\hline Co & 2 & 2 & $4 \times 10^{-5}$ & (Komasawa et al., 1981) & 0.35 \\
Zn & 2 & 1.5 & $0.945 \times 10^{-2}\left(\mathrm{~mol} / \mathrm{dm}^{3}\right)^{1 / 2}$ & (Huang and Juang, 1986) & 0.65 \\
Ni & 2 & 3 & $4.5 \times 10^{-5} \mathrm{dm}^{3} / \mathrm{mol}$ & (Komasawa et al., 1981) & Fig. 1 \\
\hline
\end{tabular}

not play a role in dilute limit, irrespective of the value of the equilibrium constant $K_{\mathrm{b}}$ for it.

\section{Results and discussion}

Quantitative predictions for the proposed mechanism require equilibrium constants $K_{i}$ and $K_{\mathrm{b}}$ to be estimated. Eqs. (18)-(21) show that as $L \rightarrow 0, K_{\mathrm{a}}$ approaches $K_{i}$, irrespective of the value of $K_{\mathrm{b}}$. The value of $K_{i}$ and $q$ are therefore taken to be the same as the literature reported value of $K_{\mathrm{a}}$ and $q$ at low loading conditions. For the extraction of $\mathrm{Zn}$, these are taken to be $0.945 \times 10^{-2} \mathrm{M}^{1 / 2}$ and 1.5 , respectively, as reported by Huang and Juang (1986).

Following the procedure of Tulasi and Kumar (1999), the value of $K_{\mathrm{b}}$ can be obtained by plotting $[\overline{\mathrm{B}}] \cdot[\overline{\mathrm{D}}]$ vs. $[\overline{\mathrm{C}}]$ and taking the intercept of the best fit line of slope 2 as the value of $K_{\mathrm{b}}$. The concentrations $[\overline{\mathrm{B}}] \cdot[\overline{\mathrm{C}}]$, and $[\overline{\mathrm{D}}]$, which cannot be measured directly, were obtained by Tulasi and Kumar using mass balance equations in conjunction with the detailed experimental data on initial and equilibrium concentrations of solute, initial concentration of extractant, and equilibrium concentration of hydrogen ions. Unfortunately, such detailed data for extraction of metal ions at high loading ratio are not available in the literature; the literature reported data are mostly in the form of $C_{\text {predicted }}$ vs. $C_{\text {experimental }}$ (Mansur et al., 2002), or $K_{\text {a }}$ vs. $L$ plots (Huang and Juang, 1986). The value of $K_{\mathrm{b}}$ is therefore taken to be the best fit value for $K_{\mathrm{a}}$ vs. $L$ data, and is estimated to be 0.65 for the experimental data of Huang and Juang (1986). The agreement between the predictions and the experimental data, shown in Fig. 1, is quite good.

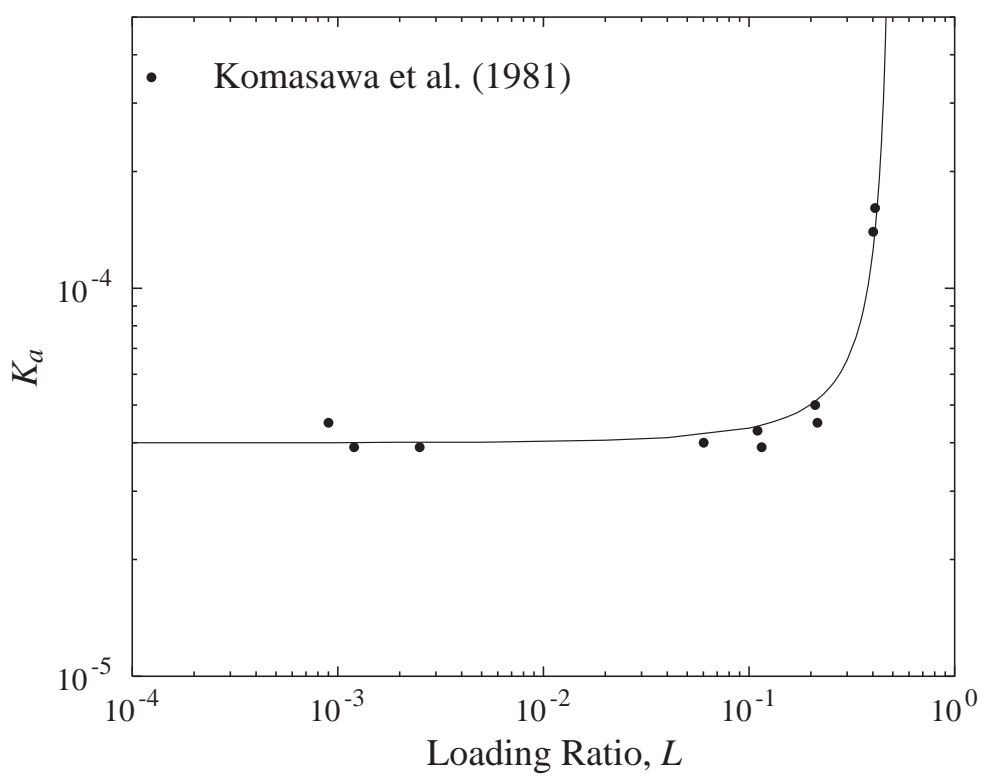

Fig. 2. Apparent equilibrium constant vs. loading ratio $L$ for Co-D2EHPA system for the data of Komasawa et al. (1981), $m=2, q=2$, $K_{i}=4 \times 10^{-5}, K_{\mathrm{b}}=0.35$. 


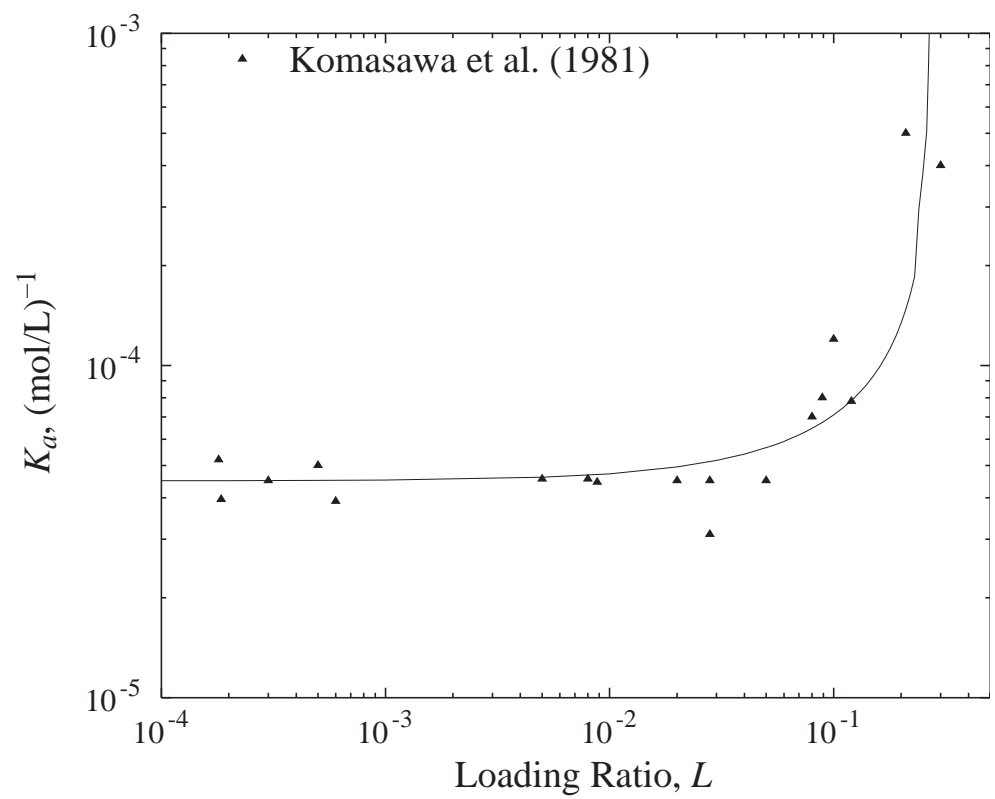

Fig. 3. Apparent equilibrium constant vs. loading ratio $L$ for Ni-D2EHPA system for the data of Komasawa et al. (1981), $m=2, q=3$ $K_{i}=4.5 \times 10^{-5} \mathrm{M}^{-1}, K_{\mathrm{b}}=2.5$.

Table 2 shows values of $m, q, K_{i}$, and $K_{\mathrm{b}}$ used to predict variation of $K_{\mathrm{a}}$ vs. $L$ for D2EHPA mediated extraction of $\mathrm{Zn}^{2+}$ and two other metals, $\mathrm{Co}^{2+}$ and $\mathrm{Ni}^{2+}$. A comparison of experimental and predicted variation of $K_{\mathrm{a}}$ vs. $L$ for the other two systems is shown in Figs. 2 and 3. The good agreement obtained for all the three systems with only one fitted parameter for each system, as $K_{i}$ values are taken from the literature (shown in Table 2), lends support to the aggregation based mechanism proposed in this work. The best fit value of $K_{\mathrm{b}}$ used to obtain these predictions was obtained by plotting $K_{\mathrm{a}}$ vs. $L$ for several values of $K_{\mathrm{b}}$ and then selecting the one which fits the data best visually, capturing the mean behaviour. A least square fit runs into difficulty because $K_{\mathrm{a}}$ approaches infinity for a finite value of $L$ (equal to $1 / q$ ) for each case. Availability of detailed experimental data for the experiments reported in the literature would have been useful in determining the $K_{\mathrm{b}}$ independently, as was the case with the D2EHPA mediated extraction of amino acids (Tulasi and Kumar, 1999). A method for calculating equilibrium extraction for the present mechanism for given values of $K_{i}, K_{\mathrm{b}}, q, m[\overline{\mathrm{B}}]_{\mathrm{i}}$, initial concentration of metal in aqueous phase, and $\mathrm{pH}$ at equilibrium is provided in Appendix.

\section{Aggregation vs. breakup}

In this section, we discuss the central issue of breakup based mechanism (Thakur, 1998; Mansur et al., 2002; Morais and Mansur, 2004) vs. aggregation based mechanism (present work) in the light of their ability to explain the dependence of $K_{\mathrm{a}}$ on $L$ alone, variation of viscosity, and other features. A derivation similar to that presented in the previous section yields for the breakup based mechanism of Mansur et al.

$$
\begin{aligned}
r=1 & -\frac{K_{i}}{K_{\mathrm{a}}} \cdot \frac{q L r^{q}}{(1-q L)^{q}} \\
- & \frac{K_{\mathrm{b}}}{\sqrt{[\bar{B}]_{i}}} \cdot \frac{K_{i}}{K_{\mathrm{a}}} \cdot \frac{\left(q-\frac{1}{2}\right) L r^{q}}{(1-q L)^{q} \sqrt{r}} \\
K_{\mathrm{a}}= & K_{i} \cdot \frac{r^{q}}{(1-q L)^{q}} \\
& +K_{i} \cdot \frac{K_{\mathrm{b}}}{\sqrt{[\bar{B}]_{i}}} \cdot \frac{r^{q}}{(1-q L)^{q} \sqrt{r}}
\end{aligned}
$$

$K_{\mathrm{b}}$ now represents equilibrium constant for the breakup reaction and $\overline{\mathrm{D}}$ represents smaller complex $\mathrm{Zn} R_{2}$ formed upon breakup of $\overline{\mathrm{C}}$. All other symbols remain 
unchanged in their meaning. As $[\overline{\mathrm{B}}]_{\mathrm{i}}$ additionally appears in Eqs. (22) and (23), one expects $K_{\mathrm{a}}$ for the breakup based mechanism to depend on $L$ and $[\overline{\mathrm{B}}]_{\mathrm{i}}$ both, unlike the aggregation based mechanism which results in dependence of $K_{\mathrm{a}}$ on $L$ alone.

Mansur et al. have estimated values of both the parameters of their mechanism $\left(K_{i}\right.$ and $\left.K_{\mathrm{b}}\right)$ simultaneously. Excluded from the experimental data used for parameter estimation was the equilibrium data in which initial concentration of extractant exceeded initial concentration of metal ion by more than twenty times. The best fit values of the parameters for $\mathrm{Zn}-$ D2EHPA system, as reported by them, are 0.298 and $0.67 \times 10^{-2} \mathrm{M}^{1 / 2}$, respectively. The experiments conducted at low values of $L$, for an unambiguous investigation into interfacial reaction, result in $K_{i}$ values of $0.945 \times 10^{-2} \mathrm{M}^{1 / 2}$ for kerosene as diluent (Huang and Juang, 1986) and $0.735 \times 10^{-2} \mathrm{M}^{1 / 2}$ for heptane as diluent (Ajawin et al., 1983). These values are 3040 times smaller than the value of $K_{i}$ reported by Mansur et al.

Fig. 4 shows variation of $K_{\mathrm{a}}$ with $L$ for three values of $[\overline{\mathrm{B}}]_{\mathrm{i}}$ for parameters of Mansur et al. The prediction shown earlier in Fig. 1 for the aggregation based mechanism is referred for a comparison. The figure shows that $K_{\mathrm{a}}$ in dilute limit is nearly unaffected by $[\overline{\mathrm{B}}]_{\mathrm{i}}$ and approaches $\mathrm{K}_{i}$, similar to the behaviour of $K_{\mathrm{a}}$ for the aggregation based mechanism. Sainz-Diaz et al. (1996) who varied $[\overline{\mathrm{B}}]_{\mathrm{i}}$ in this range also recon- firmed that interfacial reaction alone can explain the experimental data in dilute limit. The large difference between the $K_{\mathrm{a}}$ values predicted for the two mechanisms shows that the breakup based mechanism with parameters of Mansur et al. cannot explain the observations of Huang and Juang, Ajawin et al., and SainzDiaz et al. Fig. 2 of Mansur et al. shows that low percentage extraction data, some of which are likely to be in dilute limit, are also in significant error, exceeding ten times the average relative error of $11 \%$ in some cases. Thus, the breakup based mechanism with parameters of Mansur et al. although reduces to interfacial reaction controlled behaviour in the dilute limit, over-predicts the experimental data reported in this limit by more than an order of magnitude.

Variation of non-dimensional concentrations of $\overline{\mathrm{B}}$, $\overline{\mathrm{C}}$, and $\overline{\mathrm{D}}$ with $L$ for one value of $[\overline{\mathrm{B}}]_{\mathrm{i}}$ for this mechanism is also shown in Fig. 4. It shows that in the wide range of $0-0.60,[\overline{\mathrm{C}}]$ increases linearly as $[\overline{\mathrm{C}}]=\mathrm{L}$ and $[\overline{\mathrm{D}}]$ builds up only very slightly, indicating that in this range nearly all the metal is present as complex $\overline{\mathrm{C}}$ and the observed behaviour can be explained through interfacial reaction alone. At high loading, a precipitous linear decrease in $[\overline{\mathrm{C}}]$ (given by $[\tilde{\bar{C}}]=2-2 L)$ and a corresponding increase in $[\overline{\mathrm{D}}]$ (given by $[\tilde{\bar{D}}]=3 L-2$ follows. It is tempting to attribute this behaviour to increased breakup of $\overline{\mathrm{C}}$ at around $L=0.60$. This is however erroneous as the likelihood of a molecule to break, a first order process,

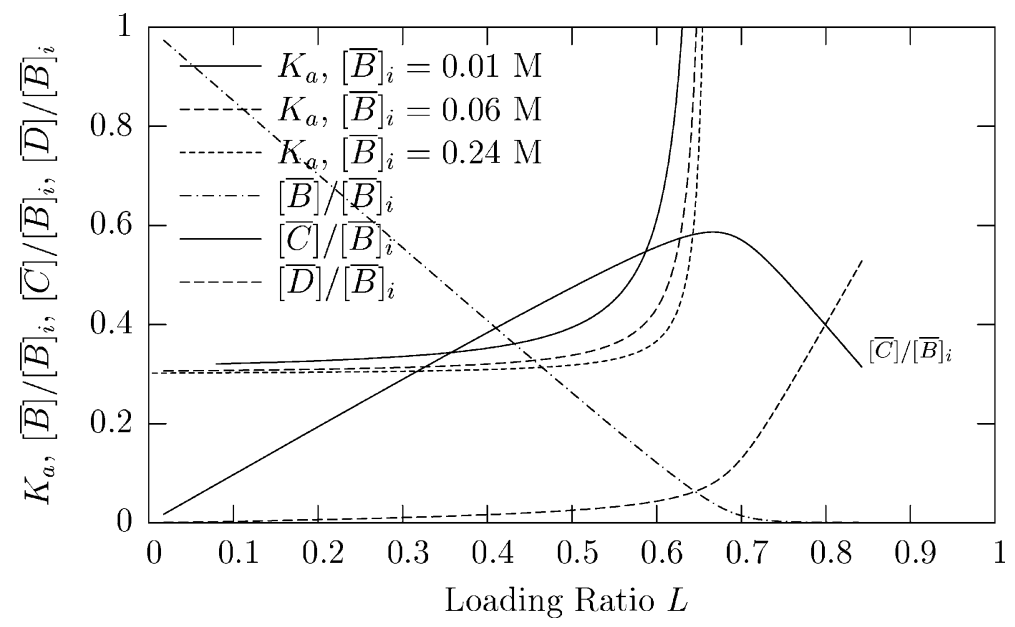

Fig. 4. Predictions of the breakup based mechanism of Mansur et al. (2002) for their for Zn-D2EHPA system $\left(K_{i}=0.298 \mathrm{M}^{1 / 2}\right.$, $K_{\mathrm{b}}=0.67 \times 10^{-2} \mathrm{M}^{1 / 2}$ ): apparent equilibrium constant $K_{\mathrm{a}}$ vs. loading ratio $L$ for various values of initial concentration of D2EHPA; variation of concentrations of various species with loading ratio for $[\overline{\mathrm{B}}]_{\mathrm{i}} 0.06 \mathrm{M}$. 
does not depend on its concentration. The reason for the above behaviour, including the wide range of $L$ in which the reaction in bulk plays no role, is the very low value fitted for $K_{b}$. The latter requires near total consumption of free extractant $\bar{B}$ for $[\bar{D}]$ to become appreciable, also shown by the profiles presented in the figure. The limiting solutions shown in the parenthesis above for $[\tilde{\bar{C}}]$ and $[\tilde{\bar{D}}]$, which match the exact predictions exceedingly well are very simply obtained by setting $[\bar{B}]=0$ for $L>1 / q$.

In order to compare the two mechanisms in a consistent manner, we have evaluated the parameters needed for the breakup based mechanism to predict the experimental data of Huang and Juang which were used earlier to estimate parameters for aggregation based mechanism. The new parameters are estimated to be: $K_{i}=0.131 \times 10^{-2} \quad \mathrm{M}^{1 / 2}$ and $K_{\mathrm{b}}=1.522 \mathrm{M}^{1 / 2}$ (for $[\overline{\mathrm{B}}]_{\mathrm{i}}=0.06 \mathrm{M}$ ). The predictions obtained with the new parameters are shown in Fig. 5. It shows variation of $K_{\mathrm{a}}$ vs. $L$ for three values of $[\overline{\mathrm{B}}]_{\mathrm{i}}$ and concentration profile for $[\overline{\mathrm{B}}]_{\mathrm{i}}=0.06 \mathrm{M}$. Ability of the breakup based mechanism to capture the experimental data of Huang and Juang (1986) with the new parameters is evident through the close match between $K_{\mathrm{a}}$ vs. $L$ for $[\overline{\mathrm{B}}]_{\mathrm{i}}=0.06 \mathrm{M}$ and the curve overlapping it, which is the same as that shown in Fig. 1. Fig. 5 shows that in limit $L \rightarrow 0, K_{\mathrm{a}}$ for three $[\overline{\mathrm{B}}]_{\mathrm{i}}$ 's differ from each other and also do not approach $K_{i}$. The mag- nitudes of non-dimensional concentrations and their variation with $L$ for the new parameters are also quite different from those for the parameters of Mansur et al. Concentration of $\bar{D}$ is an order of magnitude larger than that of $\overline{\mathrm{C}}$ even for $L \rightarrow 0$. This is quite opposite to the expectation that breakup of $\overline{\mathrm{C}}$ to form $\overline{\mathrm{D}}$ occurs at high loading. Interestingly, negligible role of breakup reaction in the dilute limit, seen earlier in Fig. 4 for the parameters of Mansur et al., is predicted to occur with the new parameters only when $[\bar{B}]_{i}$ is in excess of $20 \mathrm{M}$, a value which cannot be realized as it exceeds the concentration of pure D2EHPA itself.

Fig. 6 shows the concentrations of the same species for the aggregation based mechanism proposed in this work. The concentration of complex $\overline{\mathrm{D}}$ formed by aggregation remains close to zero at low loading ratio and becomes significant only for loading ratios larger than 0.3. The figure shows that, unlike the breakage based mechanism, the ratio of $[\overline{\mathrm{D}}] /[\overline{\mathrm{C}}]$ increases monotonically, from a starting value of 0 at $L \rightarrow 0$. As $L \rightarrow 1,[\tilde{\bar{D}}]$ approaches unity for breakage based mechanisms. In comparison, $[\tilde{\bar{D}}]$ for aggregation based mechanism approaches only 0.5 . This is because every $\overline{\mathrm{D}}$ formed by aggregation has two metal ions in it.

We have simplified final equations for breakup based mechanism (Eqs. (22) and (23)) in the limit $L \rightarrow 0$ to understand the widely different behaviours

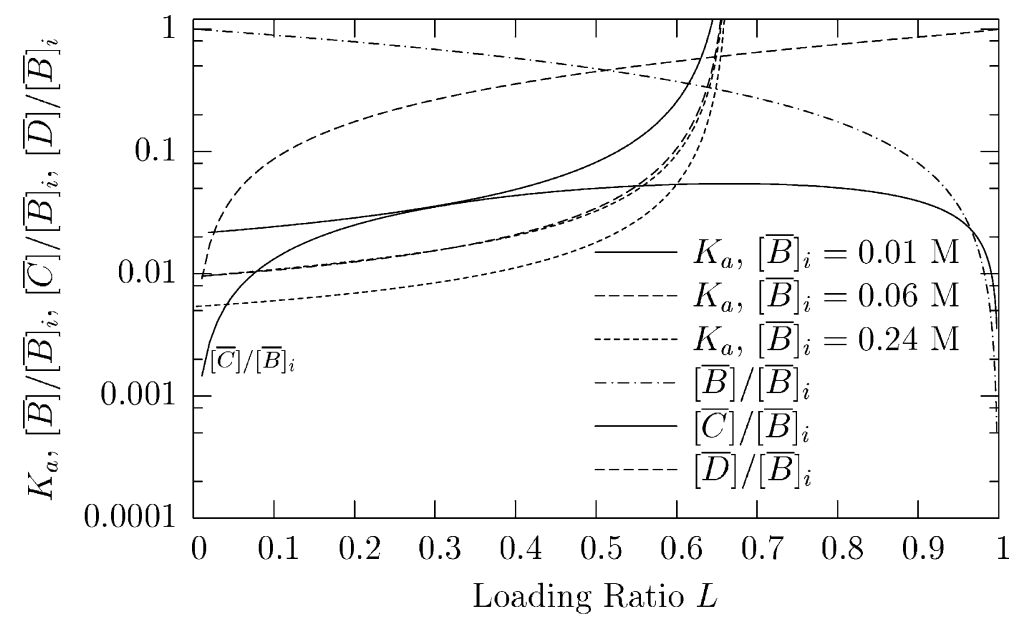

Fig. 5. Predictions of the breakup based mechanism of Mansur et al. (2002) for the parameters estimated in this work for Zn-D2EHPA system $\left(K_{i}=0.131 \times 10^{-2} \mathrm{M}^{1 / 2}, K_{\mathrm{b}}=1.522 \mathrm{M}^{1 / 2}\right)$ : apparent equilibrium constant $K_{\mathrm{a}}$ vs. loading ratio $L$ for various values of initial concentration of D2EHPA; variation of concentrations of various species with loading ratio for $[\overline{\mathrm{B}}]_{\mathrm{i}}=0.06 \mathrm{M}$. 


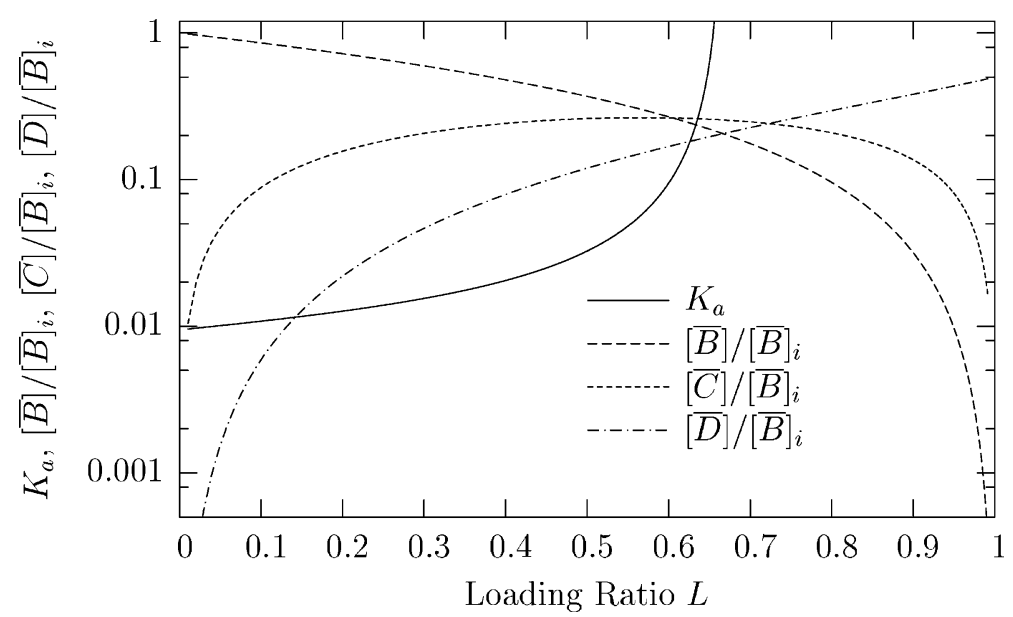

Fig. 6. Variation of $K_{\mathrm{a}}$ and non-dimensional concentration with loading ratio $L$ for the mechanism proposed in this work for $K_{i}=0.945 \times 10^{-2}$ $\mathrm{M}^{1 / 2}, K_{\mathrm{b}}=0.65$.

produced by them for different parameter values. The simplified equations in this limit are:

$$
\begin{aligned}
& K_{\mathrm{a}}=K_{i}\left(1+\frac{K_{\mathrm{b}}}{\sqrt{B_{i}}}\right)+C_{k} L \\
& r=1+C_{r} L \\
& {[\tilde{\bar{C}}]=\left(1+\frac{K_{\mathrm{b}}}{\sqrt{B_{i}}}\right)^{-1}\left(1+C_{c} L\right) L} \\
& {[\tilde{\bar{D}}]=\left(1-\left(1+\frac{K_{\mathrm{b}}}{\sqrt{B_{i}}}\right)^{-1}\left(1+C_{c} L\right) L\right) L}
\end{aligned}
$$

Constants $C_{k}, C_{r}$, and $C_{c}$ are given in the Appendix. The above limiting solution, which matches well with the exact solution for $L$ in the range $0-0.3$, is quite different from the limiting solution for the aggregation based mechanism (Eqs. (18)-(21)). First, the apparent equilibrium constant $K_{\mathrm{a}}$ does not reduce to $K_{i}$ even for $L=0$. Second, $[\overline{\mathrm{C}}]$ and $[\overline{\mathrm{B}}]$ approach zero linearly. Complex $C$ therefore breaks to form complex $[\overline{\mathrm{D}}]$ to the same extent for every value of $L$ at least in the range $0-0.3$. Thus, for the lowest possible loading that can ever be achieved, $[\overline{\mathrm{D}}]$ is predicted to be present to the same relative extent.

The reason for small effect of $[\overline{\mathrm{B}}]_{\mathrm{i}}$ on $K_{\mathrm{a}}$ for the parameters of Mansur et al. is the unusually large value of $K_{i}$, which requires $K_{\mathrm{b}}$ to be small. The above limiting solution shows that for $K_{\mathrm{b}} / \sqrt{[\bar{B}]_{i}} \ll 1$, which is satisfied by the parameters of Mansur et al., ratio $[\overline{\mathrm{D}}] /[\overline{\mathrm{C}}]$ equals $K_{\mathrm{b}} / \sqrt{[\bar{B}]_{i}}$ and $K_{\mathrm{a}}$ approaches $K_{i}$. In other words, although the breakup of complex affects equilibrium behaviour even in the dilute limit, its effect is reduced when $K_{\mathrm{b}} \ll \sqrt{[\bar{B}]_{i}}$. The new values of these parameters which predict the experimental data of Huang and Juang for assumed value of $[\overline{\mathrm{B}}]_{\mathrm{i}}=0.06 \mathrm{M}$ do not satisfy the above inequality, and hence we find that breakup of complex controls the behaviour in the dilute limit as well. Consequently, $K_{\mathrm{a}}$ changes with $[\overline{\mathrm{B}}]_{\mathrm{i}}$ in the limit $L \rightarrow 0$ and does not approach $K_{i}$.

The experimental data on viscosity variation (Table 1) show that the addition of extractant up to a concentration of $0.2 \mathrm{M}$ does not change the viscosity of the diluent. If we assume that the complex $\mathrm{Zn} R_{2}$, which is similar to the extractant $(\mathrm{HR})_{2}$ in size and shape (Fig. 7), also does not increase the viscosity of the diluent, the viscosity of the organic phase for breakup based mechanism for the parameters of Mansur et al. is then predicted to go through a maximum with an increase in $L$; the maximum is predicted to be located at a loading ratio of 0.6 , corresponding to the maximum concentration of complex $\mathrm{Zn} R_{2}(\mathrm{HR})$. The addition of extractant to the loaded organic phase is predicted to increase its viscosity because of the increase in the concentration of complex $\mathrm{Zn} R_{2}(\mathrm{HR})$ through the backward reaction in the bulk. These predictions, made after accounting for the dilution 
(a)

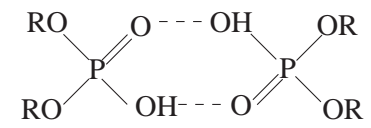

(b)

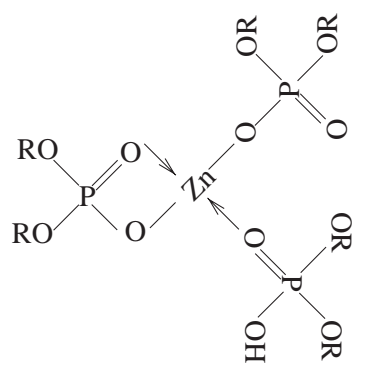

(c)<smiles>[R20][R]1([R20])O[Te]2(O1)O[PH]([R20])([R20])O2</smiles>

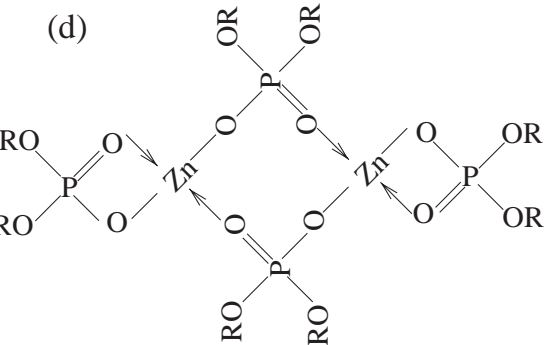

Fig. 7. Structures of various species: (a) dimer of D2EHPA, (b) complex formed on the structure $\mathrm{Zn} R_{2}(\mathrm{HR})$, (c) complex formed by breakup of that in (b) as per Mansur et al. (2002) $\mathrm{Zn} R_{2}$, (d) complex proposed in the present work formed by aggregation of that in (b) $\mathrm{Zn}_{2} R_{4}$.

caused by the addition of extractant to the organic phase, are opposite to the experimental observations. The same mechanism with the parameters estimated in this work leads to the prediction that viscosity can hardly change with loading ratio as the maximum value of $[\overline{\mathrm{C}}] /[\overline{\mathrm{B}}]$ is 0.05 (Fig. 5).

As the complex formed in the bulk for the aggregation based mechanism is nearly one and a half times as big as $[\overline{\mathrm{C}}]$, the viscosity of the organic phase is predicted to increase monotonically with loading ratio for concentration profiles shown in Fig. 6, even with conservative estimates, such as $\mu=\mu_{\mathrm{O}}\left(1+\eta_{\mathrm{C}}\right.$. $\left.[\overline{\mathrm{C}}]+\eta_{\mathrm{D}} \cdot[\overline{\mathrm{D}}]\right)$. Addition of the extractant $\overline{\mathrm{B}}$ to the loaded organic phase shifts the bulk reaction (Eq.
(8)) in backward direction, thereby decreasing the concentration of larger complexes, and hence the viscosity.

Sainz-Diaz et al. (1996) and Morais and Mansur (2004) have carried out FT-IR spectroscopy on the organic phase. Sainz-Diaz et al. (1996) carried out their analysis at low loading of the organic phase, and showed the stoichiometric coefficient to be 1.5 in this limit. Morais and Mansur (2004) have conducted the same measurements for the concentration of $\mathrm{Zn}$-D2EHPA complex in the range of $0-47.4$ $\mathrm{mol} / \mathrm{m}^{3}$. Their measurements show that the absorbance corresponding to $\mathrm{P}-\mathrm{O}-\mathrm{H}$ and $\mathrm{P}-\mathrm{O}-\mathrm{C}$ bonds decreases as the concentration of the complex increases from 0 to $37.2 \mathrm{~mol} / \mathrm{m}^{3}$, but remains constant over the range of $37.2-47.7 \mathrm{~mol} / \mathrm{m}^{3}$. The absorbance corresponding to $\mathrm{Zn}-\mathrm{O}$ and $\mathrm{P}=\mathrm{O} \rightarrow \mathrm{Zn}$ bonds continues to increase monotonically in the entire range. The authors interpret this data as providing support in favour of breakup of complex $\mathrm{Zn} R_{2}(\mathrm{HR})$ to $\mathrm{Zn} R_{2}$. We have drawn structure of D2EHPA and the three complexes under consideration in Fig. 7. It shows that two molecules of $\mathrm{Zn} R_{2}$ (Fig. 7c) and one molecule of bigger complex $\mathrm{Zn}_{2} R_{4}$ (Fig. 7d) offer the same number of $\mathrm{Zn}-\mathrm{O}$ and $\mathrm{P}=\mathrm{O} \rightarrow \mathrm{Zn}$ bonds. The spectroscopic data obtained therefore cannot shed light on whether the complex formed through the interfacial reaction breaks into smaller one as interpreted by Morais and Mansur (2004) and Thakur (1998) or aggregates as proposed in this work. The answer can possibly come from measurement of colligative properties such as vapour pressure or viscosity. As discussed earlier, the viscosity measurements of Kolarik and Grimm (1976) support the formation of bigger aggregates only. Our preliminary computer simulations also indicate that aggregation reactions are feasible.

We envisage that the complexes considered in the proposed mechanism further aggregate with each other to form even larger complexes which are needed to explain the phenomenal increase in the viscosity at high loading of metal (Table 1). A detailed approach is needed which considers aggregation of several forms of complexes and uses a model for determining the viscosity of loaded organic phase to completely understand these systems.

Thus, the analysis presented above and the experimental observations reported in the literature indi- 
cate that the behaviour of extraction systems where the extractant extracts more solute per molecule of the extractant at high loading of solute in the organic phase, can be explained through aggregation of complex in the organic phase and the release of extractant for increased extraction. Breakup of complexes in the organic phase which also releases free extractant for increased extraction is inconsistent with the experimental observations.

\section{Conclusions}

The present work shows that the attempts made in the literature to explain the equilibrium behaviour, which consider multiple interfacial reactions or breakup of the complex formed on the interface in the organic phase fail to explain interesting experimental observations on viscosity variation and dependence of apparent equilibrium constant on $L$ alone. A new two step reaction mechanism which considers aggregation of complex formed on the interface in the bulk is proposed to explain the observed behaviour. The proposed mechanism quantitatively explains the experimental findings that as the carrier is depleted in the organic phase, it is utilized more efficiently for extraction of metal ions, the dependence of apparent equilibrium constant on loading ratio alone, and variation in viscosity of the organic phase with increase in loading and a decrease with the addition of extractant. A comparison of the experimental data and the predictions for $(\mathrm{Co}, \mathrm{Zn}, \mathrm{Ni})-\mathrm{D} 2 \mathrm{EHPA}$ systems shows that the agreement between the model and the experiments is good. The proposed mechanism may also be useful in explaining the equilibrium behaviour of other systems where the efficiency of extraction increases with increase in loading of the solute in the extractant phase.

\section{Appendix A. Prediction of the extent of extraction at equilibrium}

Given the values of equilibrium constants and the values of $\left[M^{m+}\right]_{i},[\overline{\mathrm{B}}]$, and $\left[\mathrm{H}^{+}\right]$, and the volumes of aqueous and organic phases, $V_{\text {aq }}$ and $V_{\text {org }}$, respectively, the extent of equilibrium extraction can be predicted by solving the equations given in earlier sections. The full set of equations that needs to be solved is summarized as:

$$
\begin{aligned}
& {[\bar{M}]=[\bar{C}]+2[\bar{D}]} \\
& {[\bar{B}]=[\bar{B}]_{i}-(q[\bar{C}]+(2 q-1)[\bar{D}])} \\
& K_{i}=\frac{[\bar{C}]\left[H^{+}\right]^{m}}{\left[M^{m+}\right][\bar{B}]^{q}} \\
& K_{\mathrm{b}}=\frac{[\bar{D}][\bar{B}]}{[\bar{C}]^{2}} \\
& V_{\text {org }}[\bar{M}]=V_{\text {aq }}\left(\left[M^{m+}\right]_{i}-\left[M^{m+}\right]\right)
\end{aligned}
$$

The solution of the above five simultaneous nonlinear equations yields values for $[\overline{\mathrm{B}}],[\overline{\mathrm{C}}],[\overline{\mathrm{D}}],[\overline{\mathrm{M}}]$ and $[\mathrm{M}]$ at equilibrium. The loading ratio at equilibrium is given by $[\overline{\mathrm{M}}] /[\overline{\mathrm{B}}]_{\mathrm{i}}$. A general graphical method which obviates the need to solve a system of non-linear algebraic equations has also been developed (Tulasi, 2000).

\section{Appendix B. Values of constants appearing in Eqs. (24)-(27)}

$$
\begin{gathered}
K_{0}=K_{i}\left(1+\frac{K_{\mathrm{b}}}{\sqrt{[\bar{B}]_{i}}}\right) \\
C_{r}=-\frac{K_{i}}{K_{0}}\left(q+\frac{K_{\mathrm{b}}}{\sqrt{[\bar{B}]_{i}}}\left(q-\frac{1}{2}\right)\right)
\end{gathered}
$$

$$
C_{r}=K_{i}\left(\left(C_{r} q+q^{2}\right)+\frac{K_{\mathrm{b}}}{\sqrt{B_{i}}}\left(C_{r}\left(q-\frac{1}{2}\right)+q^{2}\right)\right)
$$

$$
C_{c}=C_{k} q+q^{2}-\frac{C_{k}}{K_{0}}
$$




\section{References}

Ajawin, L.A., Perez de Ortiz, E.S., Sawistowski, H., 1983. Extraction of zinc by di(2-ethylhexyl) phosphoric acid. Chem. Eng. Res. Des. 61, 62.

Bart, H.J., Marr, R., Scheks, J., Koncar, M., 1992. Modelling of solvent extraction equilibria of $\mathrm{Zn}(\mathrm{II})$ from sulfate solutions with bis-(2-ethylhexyl)-phosphoric acid. Hydrometallurgy 31, 13.

Brisk, M.L., McManamey, W.J., 1969. Liquid extraction of metals from sulphate solutions by alkylphos-phoric acids: 1. Equilibrium distributions of copper, cobalt, nickel with di-(2-ethylhexyl) phosphoric acid. J. Appl. Chem. 19, 103.

Huang, T.C., Juang, R.S., 1986. Extraction equilibrium of zinc from sulfate media with bis-(2-ethylhexyl) phosphoric acid. Ind. Eng. Chem. Fundam. 25, 752.

Kolarik, Z., Grimm, R., 1976. Acidic organophosphorous extractants-XXIV. The polymerization behaviour of $\mathrm{Cu}(\mathrm{II}), \mathrm{Cd}(\mathrm{II})$, $\mathrm{Zn}(\mathrm{II})$, and $\mathrm{Co}(\mathrm{II})$ complexes of di-(2-ethylhexyl) phosphoric acid in fully loaded organic phases. J. Inorg. Nucl. Chem. 38, 1721.

Komasawa, I., Otake, T., Higaki, Y., 1981. Equilibrium studies of the extraction of divalent metals from nitrate media with di(2-ethylhexyl) phosphoric acid. J. Inorg. Nucl. Chem. 43 (12), 3351.

Lo, T.C., Baird, M.H.I., Hanson, C., (Eds.), 1983. Handbook of Solvent Extraction. Wiley, New York.
Mansur, M.B., Slater, M.J., Biscaia, Jr., E.C., 2002. Equilibrium analysis of the reactive liquid-liquid test system $\mathrm{ZnSO} 4 /$ D2EHPA/ $n$-heptane. Hydrometallurgy 63, 117.

Morais, B.S., Mansur, M.B., 2004. Characterization of the reactive test system $\mathrm{ZnSO} 4 / \mathrm{D} 2 \mathrm{EHPA}$ in $n$-heptane. Hydrometallurgy $74,12$.

Nishihama, S., Hirai, T., Komasawa, I., 1999. Separation and recovery of gallium and indium from simulated zinc refinery residue by liquid-liquid extraction. Ind. Eng. Chem. Res. 38, 1032.

Sainz-Diaz, C.I., Klocker, H., Marr, R., Bart, H.-J., 1996. New approach in the modelling of the extraction equilibrium of zinc with bis-(2ethylhexyl)phosphoric acid. Hydrometallurgy $42,1$.

Slater, M.J., 1987. "The possible use of D2EHPA/metal as a liquidliquid extraction test system". Tech. rep., EFCE Working party on Distillation, Absorption and Extraction, Prague, CSSR.

Thakur, N.V., 1998. Extraction studies of base metals (Mn, Cu, $\mathrm{Co}$ and $\mathrm{Ni}$ ) using the extractant 2-ethylhexyl 2-ethylhexyl phosphoric acid PC88A. Hydrometallurgy 48, 125.

Tulasi, G.L., 2000. Extraction of amino acids using D2EHPA in hollow fiber supported liquid membrane. Ph.D. thesis, Indian Institute of Science, Bangalore, India.

Tulasi, G.L., Kumar, S., 1999. Amino acid extraction using D2EHPA. New description of equilibrium behaviour. AIChE J. 45, 2534. 